\section{Avaliação psicométrica do Questionário de Mudança Corporal para adolescentes}

\author{
Psychometric evaluation of the Body Change \\ Questionnaire for adolescents
}

\section{Evaluación psicométrica del Cuestionario sobre Cambios Físicos para adolescentes}

Juliana Fernandes Filgueiras Meireles 1 Ana Carolina Soares Amaral 2

Clara Mockdece Neves 1

Maria Aparecida Conti 3

Maria Elisa Caputo Ferreira 1
1 Universidade Federal de Juiz de Fora, Juiz de Fora, Brasil.

2 Instituto Federal de

Educação, Ciência e

Tecnologia do Sudeste de

Minas Gerais - Campus

Barbacena, Barbacena, Brasil.

3 Universidade de São Paulo,

São Paulo, Brasil.

Correspondência J. F. F. Meireles Rua Chanceler Oswaldo Aranha 326, apto. 1001, Juiz de Fora, $M G$

36016-340, Brasil. eujuly90@hotmail.com

\section{Abstract}

This study aimed to examine construct validity, internal consistency, and reproducibility of the Body Change Questionnaire (BCQ). A total of 439 female and male adolescents (13-22 years of age) were evaluated. Construct validity was assessed by exploratory factor analysis and correlation between the scores of the BCQ and the Body Shape Questionnaire (BSQ) and Scale Silhouettes (SS). Reliability was assessed through internal consistency and reproducibility using testretest and intra-class correlation. The scale presented a six-factor structure that almost entirely accounted for the instrument's subscales. Correlations for the total sample between BCQ and scores of the other questionnaires ranged from 0.37 to 0.46 . Internal consistency varied from 0.78 to 0.96 for each of the factors, and intraclass correlation was consistent with good reproducibility. The test-retest scores showed no statistically significant differences for the total sample or according to sex. The BCQ showed good psychometric qualities for Brazilian adolescents.

Body Image; Questionnaires; Validation Studies; Psychometrics

\section{Resumo}

O estudo objetivou analisar a validade de construto, consistência interna e reprodutibilidade do Questionário de Mudança Corporal (QMC). Foram avaliados 439 meninas e meninos (13 a 22 anos). A validade de construto foi avaliada por meio da análise fatorial exploratória e correlações entre os escores do QMC, Body Shape Questionnaire (BSQ) $e$ Escala de Silhuetas (ES). A confiabilidade foi acessada usando-se a consistência interna, e a reprodutibilidade pelo teste-reteste e pela correlação intraclasse. A escala apresentou estrutura fatorial composta por seis fatores, os quais representaram quase em sua totalidade as subescalas do instrumento. As correlações para a amostra total entre o QMC $e$ as outras escalas variaram de 0,37 a 0,46. A consistência interna apresentou valores entre 0,78 e 0,96 para cada um dos fatores, e a correlação intraclasse foi condizente com uma boa reprodutibilidade. Os escores do teste-reteste não apresentaram diferenças significantes para a amostra total e entre os sexos. Conclui-se que o QMC apresenta boas qualidades psicométricas para os adolescentes brasileiros.

Imagem Corporal; Questionários; Estudos de Validação; Psicometria 


\section{Introdução}

A insatisfação corporal tem acometido meninas e meninos em idades cada vez mais jovens 1,2 . Embora esteja relacionada com as preocupações com o peso e formato do corpo ${ }^{3}$, o modo pelo qual essa insatisfação é percebida para cada sexo é particular 4,5,6, assim como as diferentes estratégias de mudança corporal adotadas pelos indivíduos em busca de um corpo ideal.

Para o sexo feminino, historicamente, houve uma mudança do corpo considerado atrativo: passou-se das formas arredondadas da pré-história para uma silhueta mais magra nos dias atuais 7 . Devido à forte tendência cultural em considerar a magreza como padrão de corpo ideal e devido à dificuldade de se encaixar neste modelo, as jovens tendem a avaliar negativamente o seu próprio corpo 8,9 . Assim, as meninas insatisfeitas com o corpo frequentemente adotam estratégias de mudança corporal direcionadas à perda de peso, tais como: prática exacerbada de exercícios físicos, dietas restritivas, bem como uso de diuréticos e laxantes 9,10 .

Por sua vez, o corpo masculino considerado belo também sofreu modificações ao longo dos anos, tornando-se mais musculoso 11,12. Atualmente, é enfatizado um corpo estruturado por ombros largos, afinado na cintura, com músculos abdominais bem definidos 7 . Estudos revelam que a insatisfação corporal dos meninos pode estar relacionada tanto ao desejo de serem mais magros quanto de serem mais musculosos 3,6. Dessa forma, a utilização de esteroides anabólicos, suplementos alimentares e a prática de atividades físicas estão entre as estratégias mais adotadas pelos meninos insatisfeitos na tentativa de alcançar esse padrão corporal 11 .

Em ambos os sexos, a insatisfação corporal pode ser influenciada por diversos fatores, dentre eles o índice de massa corporal (IMC) 4,13,14, a cultura 9,10 , a idade 1,15 , o próprio sexo 13,16 , os comentários e os comportamentos de pais e amigos 17 , bem como as mensagens midiáticas sobre o corpo belo 18. Pressionados por esses fatores de influência, os adolescentes engajam em estratégias de modificação do corpo, muitas vezes prejudiciais à saúde, como é o caso do uso de esteroides, da prática excessiva de exercícios e do desenvolvimento de transtornos alimentares 19 .

Estudos têm demonstrado maior insatisfação corporal das meninas quando comparadas aos seus pares do sexo masculino 20,21,22. De acordo com Cafri \& Thompson ${ }^{12}$, esses achados podem estar relacionados ao fato de que a maioria dos instrumentos de avaliação da imagem corporal não considera as especificidades existentes entre os sexos. O principal foco de escalas e de questio- nários validados atualmente, em especial para a população brasileira, é a satisfação com o peso e com a forma corporal baseada na gordura (desejo de ser magra) 12,23,24, refletindo, na maioria das vezes, apenas as preocupações do sexo feminino. Entretanto, devido às peculiaridades da imagem corporal e seus componentes em meninas e meninos, são necessários instrumentos que investiguem de forma mais ampla as estratégias para alterar o tamanho e a forma corporal, utilizadas por ambos os sexos 24 .

Existem alguns instrumentos destinados a avaliar a adoção de estratégias de modificação corporal, como a Body Modification Scale, que mensura os comportamentos de mudança corporal entre adolescentes, e a Excessive Exercise Scale 25 , que avalia os sentimentos, motivações e as características da prática de exercícios pelos adolescentes. Entretanto, o questionário mais utilizado para a avaliação da adoção de estratégias de modificação corporal entre os adolescentes é o Body Change Inventory, desenvolvido por Ricciardelli \& McCabe ${ }^{3}$, que avalia estratégias adotadas para mudança corporal com foco tanto na perda de peso quanto no aumento do tamanho muscular, refletindo as preocupações de ambos os sexos. O instrumento original foi desenvolvido em quatro etapas: na primeira, as autoras propuseram um instrumento com 60 itens, com base em uma ampla revisão de literatura sobre o tema; na segunda etapa, foi realizada a análise fatorial exploratória e avaliada a consistência interna, por meio do alfa de Cronbach; o terceiro estudo correspondeu à análise fatorial confirmatória do instrumento em sua versão reduzida, utilizando apenas três das seis subescalas desenvolvidas anteriormente; já no quarto estudo, as autoras avaliaram a validade convergente do instrumento, por intermédio de sua associação a escalas de avaliação dos sintomas de transtornos alimentares 3 .

Vale destacar que o questionário original foi desenvolvido para meninos e meninas australianos, e tem sido utilizado em pesquisas que buscam investigar as formas de mudança corporal em amostras de adolescentes de diversos países, tais como: Fiji, Tonga e Austrália 26, Chile 27, China 18 e Malásia 28. Em todas as versões internacionais, o instrumento apresentou boa consistência interna. No Brasil, o Body Change Inventory encontra-se traduzido e parcialmente adaptado para a língua portuguesa 29 . O processo envolveu as etapas de tradução do questionário para o idioma português; retrotradução para o inglês; avaliação da equivalência semântica; e análise da compreensão verbal tanto por especialistas quanto por jovens brasileiros 29. Porém, os autores alertam para a necessidade de se 
avaliar suas qualidades psicométricas para que esse instrumento seja considerado apto para o seu uso.

Baseando-se no exposto, o presente estudo objetivou analisar a validade de construto (estrutura fatorial e validade convergente), a consistência interna e a reprodutibilidade (teste-reteste) do Questionário de Mudança Corporal (QMC) para adolescentes brasileiros.

\section{Métodos}

\section{Participantes}

Participaram deste trabalho 530 adolescentes, com idades entre 13 e 22 anos, estudantes de duas escolas públicas da cidade de Juiz de Fora, Minas Gerais, Brasil. Desse total, 51 alunos foram excluídos por não devolverem o Termo de Consentimento Livre e Esclarecido (TCLE) assinado pelo responsável e 40 foram excluídos por não responderem a todas as questões dos questionários. Não houve diferença nas variáveis estudadas para os alunos que compuseram a amostra final e aqueles que foram excluídos do estudo. Dessa forma, a amostra total da presente pesquisa foi de 439 participantes, sendo 189 meninos e 245 meninas, com idades de 15,92 $\pm 1,58$ e 15,91 $\pm 1,66$ anos, respectivamente. O IMC derivado do peso e estatura autorreferidos foi de $21,48 \pm 3,68$ e $21,46 \pm 3,70$ para meninas e meninos, respectivamente.

Para avaliação da reprodutibilidade, seguiram-se as recomendações de Thompson 30, as quais indicam a necessidade de uma amostra de 30 a 50 participantes. Nesta etapa foram selecionados, aleatoriamente, entre o total de participantes, 51 adolescentes, 24 meninos e 27 meninas, com média de idades de 17,29 $\pm 1,33$ e $16,78 \pm 1,12$, respectivamente.

\section{Instrumentos}

\section{- Questionário de Mudança Corporal}

Este instrumento foi desenvolvido originalmente por Ricciardelli \& McCabe 3 , a fim de avaliar a adoção de estratégias para a modificação do corpo. É composto por 48 itens em escala Likert de pontos, com suas respostas podendo variar de 1 (Nunca) a 5 (Sempre), buscando identificar a frequência de adoção de comportamentos de mudança corporal. Suas questões são organizadas em seis subescalas, a saber: Hábitos Alimentares (9 itens), Suplementos Alimentares (9 itens), Estratégias para Perder Peso (9 itens), Estratégias para Aumento do Peso (9 itens), Estraté- gias para Aumento do Tônus Muscular (6 itens) e Estratégias para Aumento do Tamanho Muscular (6 itens). Na estrutura original do instrumento essas escalas foram agrupadas em três fatores distintos: "Estratégias para redução do tamanho corporal", "Estratégias para aumento do tamanho corporal" e "Estratégias para aumento do tamanho muscular". O escore final é calculado pela soma de todas as respostas, sendo que quanto maior seu valor maior a frequência de adoção de estratégias de modificação corporal. Além disso, os escores de cada subescala também podem ser utilizados de forma independente. Utilizou-se neste estudo a versão adaptada para a população brasileira proposta por Conti et al. 29 .

\section{- Body Shape Questionnaire}

O Body Shape Questionnaire (BSQ) avalia o grau de insatisfação corporal com base em 34 itens em escala Likert de pontos, com suas respostas podendo variar de 1 (Nunca) a 6 (Sempre). Esse instrumento foi desenvolvido originalmente por Cooper et al. 31 e validado para adolescentes brasileiros por Conti et al. 23 . A pontuação varia de 34 a 204 pontos, sendo que quanto maior o escore obtido maior a insatisfação com o corpo. Neste trabalho, esse instrumento foi utilizado a fim de verificar a validade convergente do QMC por meio de sua associação à insatisfação corporal, visto serem dois comportamentos fortemente relacionados 18. Para a amostra do presente estudo, foram encontrados coeficientes alfa de Cronbach de 0,95 e 0,96 para meninos e meninas, respectivamente.

\section{- Escala de Silhuetas}

A Escala de Silhuetas (ES) de Thompson \& Gray ${ }^{32}$ consiste em uma série de figuras com uma ligeira graduação entre uma figura e a seguinte, aumentando gradativamente o padrão. Essa escala é composta por 18 figuras, sendo 9 femininas e 9 masculinas, que vão da silhueta mais magra para a mais obesa. Os participantes responderam às seguintes questões: "Escolha uma figura que melhor lhe representa no momento" e "Escolha uma única figura que representa a forma que gostaria de ter/ser". O escore é calculado pela diferença entre a silhueta escolhida como ideal e a atual, variando de -8 a 8 . Valores positivos indicam que o sujeito deseja ter uma silhueta menor que a atual e, em contrapartida, valores negativos indicam desejo de aumentar a silhueta. Dessa forma, esses valores foram utilizados como uma segunda medida para verificar a validade convergente com o QMC, correlacionando os itens direciona- 
dos à "Perda de peso" e "Aumento do peso/tamanho corporal" ao escore de insatisfação avaliado usando-se a ES.

\section{Procedimentos}

Inicialmente, foram selecionadas por conveniência duas escolas públicas da cidade de Juiz de Fora. As direções das escolas concordaram com a participação e os pesquisadores explicaram os objetivos e os procedimentos do projeto. Foram eleitas as turmas para coletas de dados de acordo com a faixa etária. Todos os alunos presentes nas aulas nos dias de coleta foram informados sobre os procedimentos necessários para a realização da pesquisa e receberam o TCLE para que os responsáveis pudessem assiná-lo. A devolução do mesmo aconteceu no dia seguinte ao primeiro encontro com os estudantes.

Os dados foram coletados entre os meses de novembro e dezembro de 2012, em sala de aula. Após a entrega do TCLE devidamente assinado pelos responsáveis, os alunos responderam de forma voluntária e individualmente aos questionários e a um cabeçalho de identificação. Os questionários foram aplicados por um mesmo pesquisador, que padronizou as orientações verbais. Os sujeitos do estudo não se comunicaram entre si e não houve limite de tempo para preenchimento dos instrumentos.

No cabeçalho, os adolescentes foram convidados a fornecer seu peso e estatura autorreferidos, a fim de calcular o IMC. Estudos populacionais e de imagem corporal têm utilizado essa forma de obtenção, destacando que não há diferenças significativas entre os valores reais e os valores autorreferidos 33 .

Foram selecionadas, entre a amostra total, três turmas para a análise da reprodutibilidade, por meio da aplicação do questionário em dois pontos no tempo, com intervalo de duas semanas entre os momentos 1 e 2 .

\section{Análise estatística}

Inicialmente, os dados foram analisados descritivamente para fins de caracterização da amostra (média, valores mínimos e máximos, desvio padrão). A avaliação psicométrica do QMC deu-se pela validade de construto, analisada por meio da análise fatorial exploratória (AFE) e pela correlação entre os escores do QMC à insatisfação corporal, avaliada pelo BSQ e pela ES. A confiabilidade foi estimada por meio da consistência interna (alfa de Cronbach) e a reprodutibilidade pela comparação dos escores do teste e do reteste e do coeficiente de correlação intraclasse. Ademais, foi realizado o teste de curtose e assimetria, que demonstrou violação da normalidade multivariada $(\mathrm{p}<0,0001)$.

Para avaliação da estrutura fatorial, realizouse a análise fatorial comum (principal axis factor), que é indicada no caso de dados que não apresentam distribuição normal. Utilizou-se, ainda, o método de rotação oblíqua promax, caracterizado por considerar a existência de correlação entre os fatores. A matriz de correlação usada foi do tipo heterogênea (two step), que reflete a integração entre as correlações policóricas (duas variáveis ordinais), polisseriais (uma variável ordinal e outra contínua) e de Pearson (duas variáveis contínuas) em uma única matriz 34 . No caso deste estudo, por serem derivados de uma escala Likert, a matriz gerada foi baseada nas correlações policóricas entre as variáveis.

Para determinação do número de fatores retidos utilizou-se a análise paralela, que é recomendada como o melhor método para acessaro número real de fatores 35 . Segundo Silva Junior et al. 36, a análise paralela gera uma amostra randômica e compara seus autovalores aos da amostra empírica. Os fatores retidos na análise são aqueles que possuem autovalor maior na amostra empírica que na amostra aleatória randômica. Para fins de aglutinação dos itens, consideraram-se significativas as cargas fatoriais superiores a 0,40 37 .

A fim de testar a qualidade de ajustamento do modelo foram calculados os índices de ajustamento goodness-of-fit index (GFI), para os quais são esperados valores superiores a 0,9 , sendo os valores acima de 0,95 considerados muito bons; e root mean square residual (RMSR), cujo valor de referência é inferior a 0,1, e os valores menores que 0,05 considerados excelentes 35 .

Por serem derivados de variáveis ordinais, os escores do QMC foram correlacionados aos do BSQ por meio do coeficiente de correlação de Spearman adotando nível de significância de 99\% ( $\mathrm{p}<0,001$ ). Para correlação aos escores de insatisfação oriundos da ES, dividiu-se os itens do QMC com base na direção de seu comportamento: redução do peso corporal (itens 1 a $13 \mathrm{e}$ 19 a 27) e aumento do peso/tamanho corporal (itens 14 a 18 e 28 a 48). Após, calculou-se o coeficiente de correlação de Spearman entre esses dois escores e a insatisfação corporal avaliada pela ES $(\mathrm{p}<0,001)$.

A confiabilidade foi avaliada usando-se o coeficiente alfa de Cronbach e de seu correspondente ordinal. Para ambas as medidas, quanto mais próximo de 1 maior a consistência interna do instrumento 35 .

Além disso, para avaliação da reprodutibilidade realizou-se o teste de Wilcoxon-Mann-Whitney para amostras pareadas. Para essa medida, as médias dos escores obtidos pelos participan- 
tes nos momentos 1 e 2 foram comparadas e calculou-se o coeficiente de correlação intraclasse.

Para as análises de estatística descritiva, inferencial e para a AFE, utilizou-se o software Statistical Package for the Social Sciences (SPSS) versão 21.0 (IBM Corp., Armonk, Estados Unidos) e seu Menu-R (R versão 2.14.2; The R Foundation for Statistical Computing, Viena, Áustria; http:// www.r-project.org).

Vale ressaltar que o projeto do presente trabalho foi aprovado pelo Comitê de Ética e Pesquisa do Hospital das Clínicas da Faculdade de Medicina da Universidade de São Paulo (protocolo 0586/08) e sua execução está de acordo com a Resolução no 466/12 do Conselho Nacional de Saúde.

\section{Resultados}

\section{Estrutura fatorial do QMC}

Inicialmente, realizaram-se os testes a fim de verificar a adequação da amostra à análise fatorial, os quais apresentaram valores satisfatórios. A análise paralela indicou a retenção de seis fatores, que explicaram $73,4 \%$ da variância.

As cargas fatoriais para cada um dos itens estão representadas na Tabela 1 . O primeiro fator, composto por 12 itens, representou a união das subescalas Estratégias para Aumento do Tônus Muscular e Estratégias para Aumento do Tamanho Muscular. O fator 2 agrupou todos os itens da subescala Estratégias para Perda de Peso. O terceiro fator apresentou os mesmos itens da subescala Hábitos Alimentares. O fator 4 agregou os itens da subescala Estratégias para Aumento do Peso. Os itens da subescala Suplementos Alimentares foram divididos nos fatores 5 e 6 , sendo o

Tabela 1

Cargas fatoriais com rotação varimax para o Questionário de Mudança Corporal (QMC); \% da variância explicada e consistência interna.

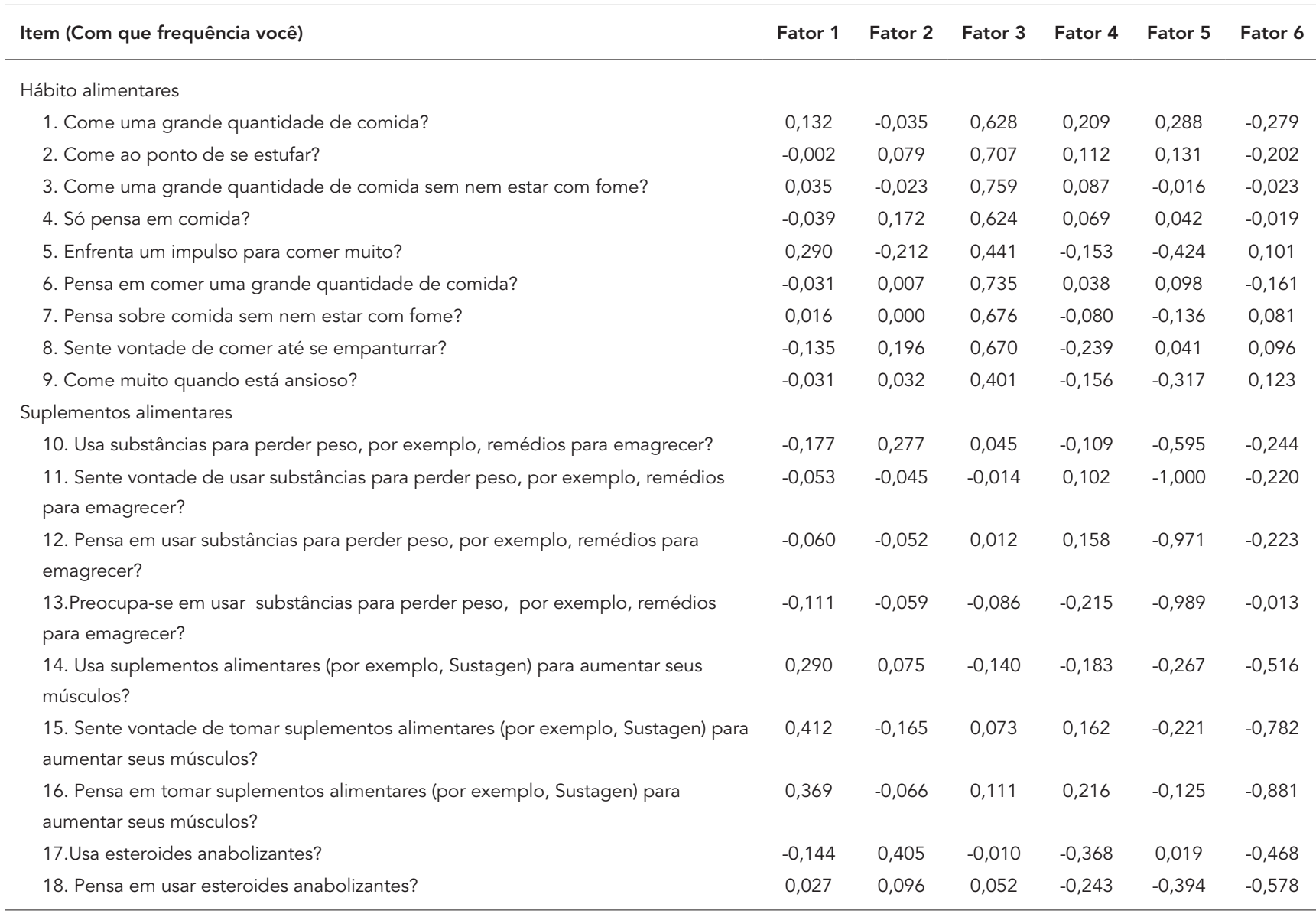

(continua) 
Tabela 1 (continuação)

Estratégias para perder peso

19. Pensa em comer menos para perder peso?

20. Come menos para perder peso?

21. Preocupa-se em comer menos para perder peso?

22. Pensa em fazer mais exercícios para perder peso?

23. Faz mais exercícios para perder peso?

24. Preocupa-se em fazer mais exercícios para perder peso?

25. Muda a quantidade de suplementos alimentares que você usa para perder peso?

26. Pensa em mudar a quantidade de suplementos alimentares que você usa para perder peso?

27. Preocupa-se em mudar a quantidade de suplementos alimentares que você

usa para perder peso?

Estratégias para aumento de peso

28. Pensa em comer mais para aumentar seu peso?

29. Come mais para aumentar seu peso?

30. Preocupa-se em comer mais para aumentar seu peso?

31. Pensa em fazer mais exercícios para aumentar seu peso?

32. Faz mais exercícios para aumentar seu peso?

33. Preocupa-se em fazer mais exercícios para aumentar seu peso?

34. Muda a quantidade de suplementos alimentares que você usa para aumentar seu peso?

35. Pensa em mudar a quantidade de suplementos alimentares que você usa para aumentar seu peso?

36. Preocupa-se em mudar a quantidade de suplementos alimentares que você usa para aumentar seu peso?

Estratégias para aumento do tônus muscular

37. Preocupa-se em mudar a sua alimentação para aumentar seu tônus muscular?

38. Muda sua alimentação para aumentar seu tônus muscular?

39. Pensa em mudar sua alimentação para aumentar seu tônus muscular?

40. Pensa em fazer mais exercícios para aumentar seu tônus muscular?

41. Faz mais exercícios físicos para aumentar seu tônus muscular?

42. Preocupa-se em fazer mais exercícios físicos para aumentar seu tônus muscular?

Estratégias para aumento do tamanho muscular

43. Preocupa-se em mudar sua alimentação para tornar seus músculos maiores?

44. Muda sua alimentação para tornar seus músculos maiores?

45. Pensa em mudar sua alimentação para tornar seus músculos maiores?

46. Pensa em fazer mais exercícios físicos para tornar seus músculos maiores?

47. Faz mais exercícios físicos para tornar seus músculos maiores?

48. Preocupa-se em fazer mais exercícios para tornar seus músculos maiores?

\% da variância explicada

Alfa de Cronbach

$\begin{array}{llllll}\text { Fator } 1 & \text { Fator } 2 & \text { Fator } 3 & \text { Fator } 4 & \text { Fator } 5 & \text { Fator } 6\end{array}$

$\begin{array}{llllll}0,159 & 0,371 & 0,078 & 0,292 & -0,347 & 0,296 \\ 0,103 & 0,497 & -0,003 & 0,205 & -0,218 & 0,220 \\ 0,138 & 0,406 & 0,086 & 0,179 & -0,309 & 0,334 \\ 0,190 & 0,672 & 0,113 & 0,343 & -0,028 & 0,148 \\ 0,312 & 0,667 & -0,096 & 0,193 & 0,011 & 0,130 \\ 0,198 & 0,697 & 0,081 & 0,202 & -0,016 & 0,110 \\ -0,149 & 1,107 & -0,011 & -0,207 & 0,175 & -0,076 \\ & & & & & \\ -0,167 & 1,014 & 0,034 & -0,177 & 0,098 & -0,084 \\ & & & & & \\ -0,134 & 1,015 & 0,065 & -0,130 & 0,098 & -0,135 \\ & & & & & \\ 0,071 & 0,173 & -0,148 & -0,901 & 0,004 & -0,051 \\ 0,046 & -0,147 & 0,199 & -0,769 & 0,137 & 0,073 \\ -0,001 & -0,127 & 0,268 & -0,766 & 0,147 & 0,073 \\ 0,002 & -0,143 & 0,177 & -0,910 & 0,035 & 0,202 \\ 0,273 & -0,125 & 0,055 & -0,742 & 0,005 & 0,066 \\ 0,215 & 0,004 & 0,026 & -0,814 & -0,012 & 0,017 \\ 0,199 & -0,019 & 0,040 & -0,854 & 0,014 & 0,076 \\ 0,140 & -0,144 & -0,986 & -0,109 & 0,031 \\ & 0,104 & -0,133 & -0,949 & -0,075 & -0,005\end{array}$

$\begin{array}{cccccc}0,847 & -0,039 & 0,010 & 0,017 & 0,028 & -0,112 \\ 0,819 & 0,139 & -0,015 & -0,056 & 0,084 & -0,061 \\ 0,822 & 0,064 & 0,080 & 0,051 & 0,063 & -0,162 \\ 0,873 & -0,031 & 0,036 & 0,158 & 0,037 & -0,160 \\ 0,834 & 0,079 & -0,071 & 0,013 & 0,103 & -0,107 \\ 0,857 & -0,035 & -0,058 & -0,001 & -0,090 & -0,111 \\ & & & & & \\ 0,832 & -0,067 & -0,010 & -0,152 & 0,020 & -0,034 \\ 0,802 & 0,064 & -0,114 & -0,299 & 0,056 & 0,083 \\ 0,876 & -0,137 & 0,033 & -0,128 & -0,061 & -0,017 \\ 0,862 & -0,057 & 0,043 & -0,110 & 0,039 & -0,020 \\ 0,788 & 0,001 & -0,054 & -0,138 & 0,074 & -0,070 \\ 0,813 & 0,029 & 0,022 & -0,100 & 0,148 & -0,043 \\ 31,11 & 19,69 & 9,26 & 6,84 & 4,21 & 2,30 \\ 0,98 & 0,95 & 0,86 & 0,97 & 0,93 & 0,89\end{array}$

primeiro com as questões direcionadas à perda de peso e o segundo com as questões referentes ao uso destas substâncias para o aumento da muscularidade.

Na análise da matriz fatorial três itens apresentaram cargas cruzadas. A questão 5 apresentou carga dividida entre os fatores 3 e 5 . Já o item
15 exibiu cargas significativas nos fatores 1 e 6 . Por último, o item 17 carregou significativamente nos fatores 2 e 6 . No caso desses três itens, optou-se por mantê-los nos fatores correspondentes às suas subescalas de origem, visto serem as cargas mais elevadas: item 5 no fator 3 , item 15 no fator 6 e item 17 no fator 6 . 
Além disso, quatro itens (11, 25, 26 e 27) apresentaram cargas superiores a 1 em seus respectivos fatores, denominados Heywood Cases, os quais indicam um ajustamento limitado do modelo fatorial.

A matriz de correlação revelou associação moderada entre diversos fatores, como pode ser verificado na Tabela 2. Destacam-se as associações entre os fatores "Suplementos alimentares para perda de peso" e "Estratégias para perda de peso" $(-0,638)$ e entre os fatores "Suplementos alimentares para aumento do peso" e "Estratégias para aumento do peso" $(0,625)$.

Os índices de ajustamento para essa estrutura fatorial foram 0,98 para GFI e 0,036 para RMSR.

\section{Associação à insatisfação corporal}

O escore do QMC esteve significativamente correlacionado à insatisfação corporal avaliada por meio do BSQ ( $p<0,001)$, tanto para a amostra total quanto para os grupos estratificados segundo o sexo. Os coeficientes de correlação de Spearman indicaram correlação positiva e moderada entre os escores do QMC, tanto para a amostra total quanto para meninos e meninas $(0,46,0,52$ e 0,49 , respectivamente), indicando que quanto maior a insatisfação com o corpo maior a frequência de comportamentos de mudança corporal. Esses resultados estão descritos na Tabela 3.

De forma semelhante, os escores de insatisfação avaliados pela ES também apresentaram correlação significativa aos do QMC ( $\mathrm{p}<0,001)$. Para esta análise, optou-se por dividir os itens do questionário em duas dimensões: questões direcionadas à "Perda de peso" e para "Aumento do peso/tamanho corporal", a fim de verificar sua associação aos escores positivos e negativos derivados da ES. Os escores correspondentes aos itens direcionados à "Perda de peso" apresentaram correlação positiva com a ES $(0,41)$, indicando que pessoas que desejam reduzir a silhueta possuem escores também elevados nestes itens. Em contrapartida, os itens direcionados ao "Aumento do peso/tamanho corporal" apresentaram correlação negativa com a ES $(-0,37)$, indicando que pessoas que desejam aumentar sua silhueta apresentam este comportamento refletido também nos itens do QMC. Os valores dos coeficientes de correlação estão apresentados na Tabela 3.

\section{Consistência interna}

Os valores do alfa de Cronbach para cada um dos fatores variaram entre 0,86 e 0,98 , indicando elevada consistência interna. Esses valores estão descritos na Tabela 1.
Tabela 2

Matriz de correlação entre os fatores.

\begin{tabular}{lccccc} 
& Fator 2 & Fator 3 & Fator $\mathbf{4}$ & Fator 5 & Fator 6 \\
\hline Fator 1 & 0,221 & 0,092 & $-0,324$ & $-0,027$ & $-0,330$ \\
Fator 2 & & 0,011 & 0,231 & $-0,638$ & 0,116 \\
Fator 3 & & $-0,152$ & $-0,132$ & 0,018 \\
Fator 4 & & & $-0,346$ & 0,625 \\
Fator 5 & & & & $-0,180$ \\
\hline
\end{tabular}

\section{Reprodutibilidade}

Os escores médios obtidos nos momentos 1 e 2 não apresentaram diferença estatisticamente significativa para a amostra total $(p=0,053)$ ou para os grupos estratificados de acordo com os sexos $(\mathrm{p}=0,222$ para meninos e $\mathrm{p}=0,130$ para meninas) (Tabela 2). Os índices de correlação intraclasse também foram satisfatórios, apresentando valores superiores a 0,72 ( $p<0,001)$, conforme apresentado na Tabela 3.

\section{Discussão}

O objetivo deste trabalho foi analisar as qualidades psicométricas do QMC entre adolescentes brasileiros. Embora o instrumento possuísse uma versão traduzida, cuja equivalência semântica já havia sido avaliada 29 , faltavam evidências que atestassem suas qualidades psicométricas.

No presente estudo, a estrutura fatorial original composta por três fatores não foi replicada. Foram encontrados seis fatores, cuja aglutinação representou quase em sua totalidade as subescalas da versão brasileira do QMC 29. Conforme Sousa \& Rojjanasrirat 38 , a adaptação transcultural implica a adequação da escala à realidade de destino, sendo que variáveis como a forma de administração, o número de itens e os valores das propriedades psicométricas não necessitam ser idênticos.

Assim, os resultados deste estudo, apesar de diferirem do estudo original, representam as características da amostra avaliada. Salienta-se, entretanto, que a própria estrutura original não refletiu as subescalas na íntegra, apresentando união de algumas subescalas em um único fator ${ }^{3}$. Portanto, destaca-se que a utilização do QMC, ou de suas subescalas, em amostras brasileiras deve ser subsidiada pelos resultados do presente estudo, os quais apontam para a qualidade do instrumento adaptado para a língua portuguesa. 
Tabela 3

Valores referentes à validade convergente, consistência interna e reprodutibilidade do Questionário de Mudança Corporal (QMC) entre adolescentes.

\begin{tabular}{|c|c|c|c|c|c|c|}
\hline \multirow[t]{2}{*}{ Análise/Variável/Parâmetro } & \multicolumn{6}{|c|}{ Sujeitos } \\
\hline & Meninos & Valor de $p$ & Meninas & Valor de $p$ & Total & Valor de $p$ \\
\hline Validade convergente & $\mathrm{n}=189$ & & $n=245$ & & $n=439$ & \\
\hline \multicolumn{7}{|l|}{ QMC-BSO } \\
\hline Escore QMC [Média (DP)] & $83,77(27,62)$ & & $77,99(20,95)$ & & $80,48(24,19)$ & \\
\hline Escore BSQ [Média (DP)] & $58,39(27,06)$ & & $72,84(36,74)$ & & $66,58(33,63)$ & \\
\hline Rho & 0,52 & $<0,001$ & 0,49 & $<0,001$ & 0,46 & $<0,001$ \\
\hline \multicolumn{7}{|l|}{ QMC-ES } \\
\hline Escore "Perda de peso" [Média (DP)] & $34,09(9,49)$ & & $39,98(13,03)$ & & $37,44(11,99)$ & \\
\hline Escore ES [Média (DP)] & $-0,39(1,54)$ & & $0,69(2,06)$ & & $0,23(1,93)$ & \\
\hline Rho & 0,17 & $<0,01$ & 0,51 & $<0,001$ & 0,41 & $<0,001$ \\
\hline $\begin{array}{l}\text { Escore "Aumento de peso/tamanho } \\
\text { corporal" [Média (DP)] }\end{array}$ & $49,68(23,29)$ & & $38,01(15,77)$ & & $43,04(20,24)$ & \\
\hline Escore ES [Média (DP)] & $-0,39(1,54)$ & & $0,69(2,06)$ & & $0,23(1,93)$ & \\
\hline Rho & $-0,23$ & $<0,001$ & $-0,35$ & $<0,001$ & $-0,37$ & $<0,001$ \\
\hline Reprodutibilidade & $n=24$ & & $n=27$ & & $\mathrm{n}=51$ & \\
\hline \multicolumn{7}{|l|}{ Escores do QMC } \\
\hline Média T1 (DP) & $77,90(21,91)$ & 0,22 & $80,42(18,88)$ & 0,13 & $79,24(20,15)$ & 0,053 \\
\hline Média T2 (DP) & $73,79(27,46)$ & & $73,64(23,28)$ & & $73,10(24,86)$ & \\
\hline \multicolumn{7}{|l|}{$\mathrm{T} 1 \times \mathrm{T} 2$} \\
\hline $\mathrm{r}_{\text {icc }}$ & 0,77 & $<0,001$ & 0,72 & $<0,001$ & 0,75 & $<0,001$ \\
\hline
\end{tabular}

BSQ: Body Shape Questionnaire; DP: desvio padrão; ES: Escala de Silhuetas; T1: escores do teste; T2: escores do reteste (2 semanas após T1).

A junção das subescalas Estratégias para $A u$ mento do Tônus Muscular e Estratégias para Aumento do Tamanho Muscular no fator 1 revela que para os adolescentes brasileiros não há diferença clara entre hipertrofia (aumento do tamanho) e tonicidade (aumento do tônus) muscular, talvez até mesmo pelo desconhecimento do significado da palavra "tônus". Em função das características dos itens, esse fator foi denominado "Estratégias para aumento da musculatura (tônus e hipertrofia)". Já em relação à subescala Suplementos Alimentares, os resultados deste estudo se mostram interessantes. Essa subescala foi dividida em dois fatores, sendo que o primeiro (fator 5) diz respeito ao "Uso de suplementos para a perda de peso" e o outro (fator 6) caracteriza-se por itens relacionados ao "Uso de substâncias para aumento da muscularidade”. Dadas as características da insatisfação corporal entre meninos e meninas 39 , pode-se afirmar que esses comportamentos representam as diferentes direções do uso de substâncias para cada sexo, justificando, assim, seu agrupamento em fatores distintos. Os demais fatores refletiram na íntegra as subescalas do instrumento, com o fator 3 representando Hábitos Alimentares, o fator 2 aglutinando os itens relacionados às Estratégias para Perder Peso e o fator
4 com as questões destinadas a avaliar a adoção de Estratégias para Aumento do Peso.

Em relação às cargas fatoriais, três itens apresentaram cargas significativas em mais de um fator. Segundo Damásio 40, a presença de cargas fatoriais complexas, ou seja, que carregam em mais de um fator, é comum em medidas psicológicas. Essas devem, entretanto, ser analisadas cuidadosamente, a fim de tomar decisões que preservem a qualidade da escala. No caso do presente trabalho, optou-se pela não exclusão dos itens que apresentaram cargas cruzadas e pela manutenção dos mesmos nos fatores correspondentes às suas subescalas originais. Essa decisão foi baseada, especialmente, na avaliação do conteúdo dos itens.

Em relação à ocorrência dos Heywood $\mathrm{Ca}$ ses, Hair Júnior et al. 37 explicam que estes casos ocorrem quando a solução fatorial produz uma estimativa de variância de erro inferior a 0 , o que implicaria que mais de $100 \%$ da variância em um item é explicada. Para esses autores, isso implica um mal ajustamento do modelo fatorial. Entretanto, existem indicações de que a utilização de rotações oblíquas gera cargas fatoriais superiores a 1 , sem implicar um mal ajustamento do modelo 41 . Esse parece ser o caso do presente estudo, 
para o qual a qualidade do modelo é reforçada pelos valores obtidos para os índices de ajustamento $(\mathrm{GFI}=0,98$; $\mathrm{RMSR}=0,036)$ que indicam a qualidade do modelo fatorial proposto.

Os resultados obtidos por meio da matriz de correlação dos fatores apontam para a não existência de fatores ortogonais entre si, ou seja, a matriz de correlações oriunda das rotações oblíquas explicita a relação teorizada entre os fatores. No caso do QMC, percebe-se que os fatores mais fortemente relacionados indicam estratégias direcionadas no mesmo sentido (fatores 2 e 5 para perda de peso, e fatores 4 e 6 para aumento do peso/muscularidade).

Com relação à validade convergente, o instrumento respondeu de forma satisfatória a todas as medidas aplicadas. Os achados apontaram uma correlação moderada $(0,46)$ e significativa ( $\mathrm{p}$ $<0,001)$ para a amostra total entre os escores do QMC e do BSQ, indicando sua associação à insatisfação corporal. Dessa forma, jovens com maior insatisfação corporal tendem a apresentar maior frequência de comportamentos de mudança corporal. Para a ES, os indivíduos que desejavam diminuir a silhueta corporal apresentaram maiores escores nas subescalas relacionadas à perda de peso do QMC $(0,41 ; \mathrm{p}<0,001)$. Em contrapartida, naqueles que desejavam aumentar a silhueta, as pontuações nos itens direcionados ao aumento do peso/tamanho corporal foram maiores (-0,37; $\mathrm{p}<0,001)$. Esses resultados confirmam a associação da adoção de comportamentos de mudança corporal à insatisfação corporal 3,18, atestando a validade convergente do QMC.

Apesar do QMC já ter sido utilizado em diferentes populações 18,26,27,28, em nenhum destes estudos os autores analisaram todas as qualidades psicométricas do instrumento. Nesses foram descritos apenas os valores de consistência interna, calculada usando-se o alfa de Cronbach. No estudo de $\mathrm{Xu}$ et al. 18 , realizado na China, os dados foram apontados de acordo com cada subescala, sendo que os valores de alfa variaram de 0,76 a 0,88 . Mellor et al. 27, avaliando adolescentes do Chile, e Mellor et al. 28, em jovens da Malásia, utilizaram apenas duas subescalas do questionário para ambos os sexos, as quais também apontam adequada consistência interna, com valores variando entre 0,72 e 0,90 . O instrumento também foi utilizado em meninas e meninos de Fiji, Tonga e Austrália por McCabe et al. 26, para os quais demonstrou consistência interna de 0,75 a 0,84 . Para o presente estudo, no que diz respeito à confiabilidade do instrumento, os valores de consistência interna, avaliados usando-se o alfa de Cronbach ordinal, foram superiores a 0,86, considerados elevados 37 , indicando a consistência interna da estrutura fatorial encontrada.
Em relação à reprodutibilidade, a análise confirmou que, após um intervalo de duas semanas, não foram encontradas diferenças significativas para meninos e meninas $(\mathrm{p}=0,22 \mathrm{e} \mathrm{p}=0,13$, respectivamente), bem como foram obtidos valores elevados de correlação intraclasse $(>0,72)$. Entretanto, quando considerada a amostra total, o valor de p revela uma diferença marginalmente significante ( $p=0,053$ ), o que pode se justificar em função da sensibilidade deste tipo de análise ao tamanho da amostra, ou seja, quanto maior o n maior a probabilidade de se obter uma diferença significativa ${ }^{41}$. Ademais, dentre os estudos que utilizaram o QMC em outros países não foram verificados dados de avaliação da reprodutibilidade $18,26,27,28$.

É importante ressaltar que este estudo apresenta algumas limitações. A principal delas refere-se à validade externa do QMC. A amostra do estudo foi constituída de escolares de setor público de ensino, o que pode não refletir as características da população brasileira como um todo. Contudo, com base nos resultados encontrados sugere-se que o instrumento está apto para ser usado em amostras semelhantes à desta pesquisa. Outra possível limitação diz respeito à utilização de medidas autorreportadas para a verificação da validade convergente (BSQ e ES). Entretanto, argumenta-se que, além de serem instrumentos de fácil aplicabilidade, o BSQ e a ES têm validade confirmada entre adolescentes brasileiros 23,42. Além disso, ressalta-se que as medidas autorreportadas têm sido as mais utilizadas na avaliação da insatisfação com a imagem corporal, tanto no Brasil quanto em estudos internacionais 4,8,9,16,43.

Conclui-se que o QMC apresentou adequada validade de construto, confiabilidade e reprodutibilidade, ratificando suas características psicométricas para adolescentes brasileiros de ambos os sexos. É importante ressaltar que os resultados do presente estudo contribuem para a validação deste instrumento, devendo ser corroboradas por novas pesquisas que investiguem sua validade para outras populações, bem como a validade externa e discriminante da estrutura fatorial encontrada, além do exame detalhado das correlações residuais e das propriedades escalares. Com base nesses resultados, espera-se que este questionário possa ser utilizado para avaliar as estratégias corporais de jovens, na medida em que ele reflete as preocupações de ambos os sexos. Reforça-se a necessidade de mais estudos que avaliem os artifícios utilizados por meninas e meninos para a alteração do corpo, sejam eles para diminuir o tamanho do corpo, aumentar o tamanho corporal ou aumentar a muscularidade. 


\section{Resumen}

El objetivo del estudio fue evaluar la validez de constructo, la consistencia interna y la reproducibilidad del Cuestionario de Cambios Físicos (QMC por sus siglas en portugués). Se evaluaron a 439 niñas y niños (13-22 años). La validez de constructo se evaluó mediante análisis factorial exploratorio y las correlaciones entre las puntuaciones de la QMC, Body Shape Questionnaire (BSQ) y la Escala Silhouettes (ES). La confiabilidad se consiguió por la consistencia interna y la reproducibilidad test-retest y la correlación intraclase. La escala tiene una estructura factorial compuesta por seis factores, que representaron casi en su totalidad las subescalas del instrumento. Las correlaciones de la muestra total entre QMC y las puntuaciones de los otros de cuestionarios oscilaron 0,37-0,46. La consistencia interna varió de 0,78 y 0,96 para cada uno de los factores y la correlación intraclase fue consistente con buena reproducibilidad. Las puntuaciones de test-retest mostraron diferencias significativas para la muestra total y de género. La conclusión es que el QMC tiene buenas cualidades psicométricas en adolescentes brasileños.

Imagen Corporal; Cuestionarios; Estudios de Validación; Psicometría

\section{Colaboradores}

Todos os autores contribuíram igualmente em todas as etapas do trabalho.

\section{Referências}

1. Fidelix YL, Minatto G, Ribeiro RR, Santos KD, Petroski EL. Dados sociodemográficos, estado nutricional e maturação sexual de escolares do sexo masculino: exposição à insatisfação com a imagem corporal. Rev Educ Fís 2013; 24:83-92.

2. Smolak LM. Body image in children and adolescents: where do we go from here? Body Image 2004; 1:15-28.

3. Ricciardelli LA, McCabe MP. Psychometric evaluation of the Body Change Inventory: an assessment instrument for adolescent boys and girls. Eat Behav 2002; 3:45-59.

4. Dumith SC, Menezes AMB, Bielemann RM, Petresco S, Silva ICM, Linhares RS, et al. Insatisfação corporal em adolescentes: um estudo de base populacional. Ciênc Saúde Coletiva 2012; 17:2499-505.

5. McCabe MP, Ricciardelli LA. A longitudinal study of pubertal timing and extreme body change behaviors among adolescent boys and girls. Adolescence 2004; 39:145-66.

6. McCabe MP, Ricciardelli LA. Sociocultural influences on body image and body changes among adolescent boys and girls. J Soc Psychol 2003; 143:5-26.

7. Murnen SK. Gender and body images. In: Cash T, Smolak L, editors. Body image: a handbook of science, practice, and prevention. London: The Guilford Press; 2011. p. 173-9.

8. Alves E, Vasconcelos FAG, Calvo MCM, Neves J. Prevalência de sintomas de anorexia nervosa e insatisfação com a imagem corporal em adolescentes do sexo feminino do Município de Florianópolis, Santa Catarina, Brasil. Cad Saúde Pública 2008; 24:503-12.

9. Fortes LS, Amaral ACS, Almeida SS, Ferreira MEC. Internalização do ideal de magreza e insatisfação com a imagem corporal em meninas adolescentes. Psico (Porto Alegre) 2013; 44:432-8.

10. Rodgers R, Cabrol H, Paxton SJ. An exploration of the tripartite influence model of body dissatisfaction and disordered eating among Australian and French college women. Body Image 2011; 8 : 208-15.

11. Cafri G, Thompson JK, Ricciardelli L, McCabe M, Smolak L, Yesalis C. Pursuit of the muscular ideal: physical and psychological consequences and putative risk factors. Clin Psychol Rev 2005; 25 : 215-39.

12. Cafri G, Thompson JK. Measuring male body image: a review of the current methodology. Psychol Men Masc 2004; 5:18-29.

13. Finato S, Rech RR, Migon P, Gavineski IC, Toni V, Halpern R. Insatisfação com a imagem corporal em escolares do sexto ano da rede municipal de Caxias do Sul, no Rio Grande do Sul. Rev Paul Pediatr 2013; 31:65-70.

14. Pelegrini A, Petroski EL. The association between body dissatisfaction and nutritional status in adolescent. Hum Movement 2010; 11:51-7.

15. Conti MA, Frutuoso MFP, Gambardella AMD. Excesso de peso e insatisfação corporal em adolescentes. Rev Nutr PUCCAMP 2005; 18:491-7. 
16. Petroski EL, Pelegrini A, Glaner MF. Insatisfação corporal em adolescentes rurais e urbanos. Motricidade 2009; 5:13-25.

17. Amaral ACS, Ferreira MEC, Scagliusi FB, Costa LS, Cordas TA, Conti MA. Avaliação psicométrica da Escala de Influência dos Três Fatores (EITF). Psicol Reflex Crit 2013; 26:213-21.

18. Xu X, Mellor D, Kiehne M, Ricciardelli L, McCabe $\mathrm{MP}, \mathrm{Xu}$ Y. Body dissatisfaction, engagement in body change behaviors and sociocultural influences on body image among Chinese adolescents. Body Image 2010; 7:156-64.

19. Ricciardelli LA, McCabe MP, Holt KE, Finemore J. A biopsychosocial model for understanding body image and body change strategies among children. J Appl Dev Psychol 2003; 24:475-85.

20. Silva TAB, Ximenes RCC, Holanda MA, Melo MG, Sougey EB, Couto GBL. Frequência de comportamentos alimentares inadequados e sua relação com a insatisfação corporal em adolescentes. J Bras Psiquiatr 2012; 61:154-8.

21. Miranda VPN, Conti MA, Bastos R, Ferreira MEC. Insatisfação corporal em adolescentes brasileiros de municípios de pequeno porte de Minas Gerais. J Bras Psiquiatr 2011; 60:190-7.

22. Vilela JEM, Lamounier JA, Dellaretti Filho MA, Barros Neto JR, Horta GM. Transtornos alimentares em escolares. J Pediatr (Rio J.) 2004; 80:49-54.

23. Conti MA, Cordás TA, Latorre MRO. A study of the validity and reability of the Brazilian verson of the Body Shape Questionnaire (BSQ) among adolecents. Rev Bras Saúde Matern Infant 2009; 9:331-8.

24. Bighetti F, Santos CB, Santos JE, Ribeiro RPP. Tradução e avaliação do Eating Attitudes Teste em adolescentes do sexo feminino de Ribeirão Preto, São Paulo. J Bras Psiquiatr 2004; 53:339-46.

25. McCabe MP, Vincent MA. Development of body modification and excessive scales for adolescents. Assessment 2002; 9:131-41.

26. McCabe MP, Ricciardelli L, Waqa G, Goundar R, Fotu K. Body image and body change strategies among adolescent males and females from Fiji, Tonga and Australia. Body Image 2009; 6:299-303.

27. Mellor D, McCabe M, Ricciardelli L, Merino ME Body dissatisfaction and body change behaviors in Chile: the role of sociocultural factors. Body Image 2008; 5:205-15.

28. Mellor D, McCabe M, Ricciardelli L, Yeow J, Daliza N, Fizlee N, et al. Sociocultural influences on body dissatisfaction and body change behaviors among Malaysian adolescents. Body Image 2009; 6:121-8.

29. Conti MA, Ferreira MEC, Amaral ACS, Hearst N, Cordás TA, Scagliusi FB. Equivalência semântica da versão em português do "Body Change Inventory”. Ciênc Saúde Coletiva 2012; 17:2457-69.
30. Thompson JK. The (mis)measurement of body image: ten strategies to improve assessment for applied and research purposes. Body Image 2004; 1:7-14.

31. Cooper PJ, Taylor MJ, Cooper Z, Fairbum CG. The development and validation of the Body Shape Questionnaire. Int J Eat Disord 1987; 6:485-94.

32. Thompson MA, Gray JJ. Development and validation of a new body-image assessment scale. J Pers Assess 1995; 64:258-69.

33. Oliveira LPM, Queiroz VAO, Silva MCM, Pitangueira JCD, Costa PRF, Demétrio F. Índice de massa corporal obtido por medidas autorreferidas para a classificação do estado antropométrico de adultos: estudo de validação com residentes no Município de Salvador, Estado da Bahia, Brasil. Epidemiol Serv Saúde 2012; 21:325-32.

34. Courtney MGR. Determining the number of factors to retain in EFA: using the SPSS R-menu v2.0 to make more judicious estimations. Practical Assessment, Research \& Evaluation 2013; 18:1-14.

35. Basto M, Pereira JM. An SPSS R-menu for ordinal factor analysis. J Stat Softw 2012; 46:1-29.

36. Silva Junior SHA, Vasconcelos AGG, Griep RH, Rotenberg L. Validade e confiabilidade do índice de capacidade para o trabalho (ICT) em trabalhadores de enfermagem. Cad Saúde Pública 2011; 27:1077-87.

37. Hair Júnior JF, Black WC, Babin BJ, Anderson RE, Tatham RL. Análise multivariada de dados. $6^{\mathrm{a}}$ Ed. São Paulo: Bookman; 2009.

38. Sousa VD, Rojjanasrirat W. Translation, adaptation and validation of instruments or scales for use in cross-cultural health care research: a clear and user-friendly guideline. J Eval Clin Pract 2011; 17:268-74.

39. Miranda VPN, Neves CM, Amaral ACS, Ferreira MEC. Imagem corporal na adolescência. In: Ferreira MEC, Castro MR, Morgado FFR, organizadoras. Imagem corporal: reflexões, diretrizes e práticas de pesquisa. Juiz de Fora: Editora UFJF; 2014. p. 67-85.

40. Damásio BF. Uso da análise fatorial exploratória em psicologia. Aval Psicol 2012; 11:213-28.

41. DeVellis RF. Scale development: theory and applications. 2nd Ed. Thousand Oaks: Sage Publications; 2003.

42. Conti MA, Latorre MRDO. Estudo de validação e reprodutibilidade de uma escala de silhueta para adolescentes. Psicol Estud 2009; 14:699-706.

43. Carvalho PHB, Neves CM, Filgueiras JF, Miranda VPN, Ferreira MEC. Percepção e insatisfação corporal de bailarinas não profissionais. Motricidade 2012; 8:758-63.

Recebido em 21/Jul/2014

Versão final reapresentada em 09/Jun/2015

Aprovado em 27/Ago/2015 